\title{
A single bacterium restores the microbiome dysbiosis to protect bones from destruction in a rat model of rheumatoid arthritis
}

Hudan Pan ${ }^{1 \dagger}$, Ruijin Guo ${ }^{1,2,3 \dagger}$, Yanmei Ju ${ }^{2,3 \dagger}$, Qi Wang ${ }^{2,3,6 \dagger}$, Jie Zhu ${ }^{2,3}$, Ying Xie', Yanfang Zheng ${ }^{1,5}$, Ting Li ${ }^{1}$,

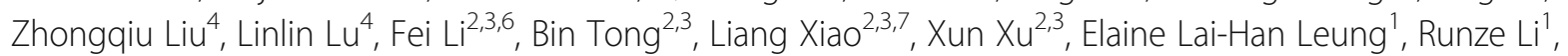
Huanming Yang ${ }^{2,3,8}$, Jian Wang ${ }^{2,3,8}$, Hua Zhou' ${ }^{1}$, Huijue Jia $^{2,3^{*}}$ and Liang Liu ${ }^{1 *}$

\begin{abstract}
Background: Early treatment is key for optimizing the therapeutic success of drugs, and the current initiating treatment that blocks the progression of bone destruction during the pre-arthritic stages remains unsatisfactory. The microbial disorder in rheumatoid arthritis (RA) patients is significantly reversed with effective treatment. Modulating aberrant gut microbiomes into a healthy state is a potential therapeutic approach for preventing bone damage.

Results: By using metagenomic shotgun sequencing and a metagenome-wide association study, we assessed the effect of Lactobacillus casei (L. casei) on the induction of arthritis as well as on the associated gut microbiota and immune disorders in adjuvant-induced arthritis (AIA) rats. Treatment of AIA rats with L. casei inhibited joint swelling, lowered arthritis scores, and prevented bone destruction. Along with the relief of arthritis symptoms, dysbiosis in the microbiome of arthritic rats was significantly reduced after $L$. casei intervention. The relative abundance of AIAdecreased Lactobacillus strains, including Lactobacillus hominis, Lactobacillus reuteri, and Lactobacillus vaginalis, were restored to normal and Lactobacillus acidophilus was upregulated by the administration of L. casei to the AIA rats. Moreover, L. casei downregulated the expression of pro-inflammatory cytokines, which are closely linked to the effect of the L. casei treatment-associated microbes. Functionally, the maintenance of the redox balance of oxidative stress was involved in the improvement in the L. casei-treated AIA rats.
\end{abstract}

Conclusion: A single bacterium, L. casei (ATCC334), was able to significantly suppress the induction of AIA and protect bones from destruction in AIA rats by restoring the microbiome dysbiosis in the gut, indicating that using probiotics may be a promising strategy for treating RA, especially in the early stage of the disease.

\section{Introduction}

Rheumatoid arthritis (RA) is a common systemic autoimmune disease associated with bone destruction [1]. Early diagnosis and treatment play pivotal roles in optimizing the therapeutic success of treatment with drugs, particularly for RA patients with high disease activity,

\footnotetext{
* Correspondence: jiahuijue@genomics.cn; lliu@must.edu.mo

${ }^{\dagger}$ Hudan Pan, Ruijin Guo, Yanmei Ju, and Qi Wang contributed equally to this work.

${ }^{2}$ BGI-Shenzhen, Shenzhen 518083, China

${ }^{1}$ State Key Laboratory of Quality Research in Chinese Medicine/Macau Institute for Applied Research in Medicine and Health, Macao University of Science and Technology, Macao, China

Full list of author information is available at the end of the article
}

presence of autoantibodies, and early joint damage [2]. Evidence is accumulating that the first few months after the onset of symptoms represent a pathologically distinct phase of the disease, offering a therapeutic window of opportunity to possibly switch off disease progression permanently [3]. However, robust diagnostic biomarkers with high sensitivity in the early stage are still lacking, and the current initiating treatment that blocks the progression of bone erosion during the pre-arthritic stages remains unsatisfactory [4].

A relationship between the gut microbiota and RA has been suspected for many years, and a number of

(c) The Author(s). 2019 Open Access This article is distributed under the terms of the Creative Commons Attribution 4.0 International License (http://creativecommons.org/licenses/by/4.0/), which permits unrestricted use, distribution, and 
epidemiological and clinical reports have implicated an array of microorganisms in RA pathogenesis. However, causation has not yet been established [5], in part because of the complex interactions between the microbiota and genetic factors such as major histocompatibility complex (MHC) molecules which may affect and modulate the composition of microbial communities [6]. Furthermore, RA-enriched gut microbial genes may act as potential molecular mimics of RAassociated antigens $[7,8]$.

We recently reported on the first catalog of the gut microbiota of Sprague-Dawley (SD) rats established by shotgun metagenomic sequencing [9]. Profiling of this catalog showed that the gut microbiome of rats compared to mice is slightly more similar to that of humans [9], and at the functional level, 97\% of the pathways are shared suggesting that rat at the functional level to a certain extent may serve as a model for humans. The microbial perturbation in RA patients was demonstrated to be partially reversed with effective treatment in our previous study [7], indicating that modulation of the gut microbiome is closely related to the effectiveness of treatment. Several clinical studies have also shown that the use of probiotics might contribute to RA remission when combined with disease-modifying anti-rheumatic drugs (DMARDs) [10]. However, the causation of changes and the dynamic changes in the gut microbiome throughout the progression of arthritis are unclear, and whether a single bacterium used as a probiotic is able to prevent or ameliorate the early stage of disease remains to be proven. Lactobacillus casei ATCC334 (L. casei) is one of the most-widely used probiotics. Mice fed with $L$. casei could significantly decrease the expression of TLR2 and TNF- $\alpha$, which were closely related to the pathogenesis of RA [11]. In the present study, we used a wellestablished RA model, adjuvant-induced arthritis (AIA), in SD rats, which is pathologically similar to human RA [12] to investigate the potential of administration of the probiotics $L$. casei for treatment of RA. We observed that administration of $L$. casei elicited profound changes in the composition of microbial species in the gut along with the changes in clinical symptoms during the processes of arthritis induction and progression.

\section{Methods}

\section{Animals}

SD rats weighing approximately $60 \mathrm{~g}$ were maintained under standard laboratory conditions at $25^{\circ} \mathrm{C}$, with a 12 $\mathrm{h} / 12 \mathrm{~h}$ light/dark cycle and 55\% humidity and fed feed and water ad libitum. The four groups with seven rats in each cage were all fed the regular (low-fat) chow. The rats were allowed 14 days to adapt to the laboratory environment before experiments were initiated. Adjuvant arthritis was induced according to the method described previously [13]. The AIA rats were randomly divided into three groups, each of seven rats, the AIA model (vehicle-treated with $0.3 \%$ sodium carboxymethyl cellulose (CMC-Na)), the L. casei-treated $\left(2 \times 10^{8}\right.$ colony-forming units (CFU)/day) group, and the methotrexate (MTX)treated $(7.6 \mathrm{mg} / \mathrm{kg} /$ week $)$ group. In addition, normal rats were used in the experiment for comparative purposes. The clinical symptoms of arthritis were evaluated through increases in hind paw volume and the arthritis score by two independent observers. Blood samples were collected from the abdominal aorta of anesthetized rats on day 30 of the experiment. Then, the rats were sacrificed by cervical dislocation. The erythrocyte sedimentation rate (ESR) was determined by a modified method based on the method selected by the International Council for Standardization in Haematology (ICSH). The levels of IL-1 $\beta$, TNF- $\alpha$, IL-17, and IL-6 in serum were determined using commercially available enzymelinked immunosorbent assay (ELISA) kits according to the manufacturer's instructions (R \& D Systems, USA).

\section{Metagenomic sequencing and annotation}

Single-end metagenomic sequencing was performed on the BGISEQ-500 platform as described previously [14]. Briefly, we constructed one DNA single-end (SE) library with a read length of $100 \mathrm{bp}$. Low-quality reads and rat reads (categorized according to an alignment with NCBI accession no. NC_005100) were removed. On average, $7.24 \mathrm{~Gb}$ of high-quality non-host sequences were obtained per sample (Additional file 15: Table S1). The relative abundances of species determined by the metagenomic sequencing were computed using the MetaPhlAn2 profiling software with default parameters [15] (Additional file 16: Table S2). Reads were mapped to the gene catalog of the SD rat gut metagenome [9] to generate the Kyoto Encyclopedia of Genes and Genomes (KEGG) ortholog and module profiles.

\section{Health and disease planes}

Health and disease planes were constructed as previously described [16]. In brief, principal coordinates analysis (PCoA) of the Bray-Curtis distance was performed at the species level, and then samples in the model or normal group were fitted to a two-dimensional plane at each time point using the least-squares method on the first three principal coordinates. The Euclidean distances from samples to these planes at each time point were calculated.

\section{Selection of arthritis-correlated microbes}

The relative abundance of the microbial species at the five TPs were used to regress to the arthritic scores. (i) Microbial species presenting in less than $5 \%$ of rats were removed. (ii) We applied log-transform to the profile of 
microbial species and subsequently normalized it by z-score. (iii) Five-fold cross-validation with ten repeats was performed on an elastic-net regularized linear regression model ( $\mathrm{R}$ 3.5.1, Glmnet2.0-16 package) using the normalized profile of microbial species against the arthritic scores. Totally, 50 regression models were obtained and we extracted arthritis-correlated species by two steps: (i) removed models in which the number of nonzero $\beta$ is less than five and (ii) selected species which is presented in more than 25 models.

\section{Fold change analysis}

The relative abundances of the selected arthritis-correlated species in the model, MTX-treated, and L. caseitreated groups were normalized by those in the normal group at each TPs and then were calculated in the $\log _{10}$ fold change (FC) compared to TP1. The change was defined as significant if a species experienced consistent $>$ $16 \mathrm{FC}\left(\log _{10} \mathrm{FC}\right.$ values are \pm 1.20$)$ in over $80 \%$ of the rats.

\section{Correlation analysis}

Semi-partial Spearman correlation tests (R 3.5.1, ppcor package 1.1) were used to calculate the correlation coefficients between the relative abundance of arthritiscorrelated microbes and the distances to HP or DP adjusted for weight and group, also between the relative abundance of identified microbes and the serum cytokines measured by commercial ELISA kit, as previously described [17], adjusted for arthritic scores, weight, and group, after removing the outliers.

\section{KEGG analysis}

Putative amino acid sequences were translated from the gene catalogs and aligned against the proteins or domains in the KEGG databases (release 79.0, with animal and plant genes removed) using BLASTP (v2.2.24, default parameter, except -e $0.01-\mathrm{b} 100-\mathrm{k} 1-\mathrm{F} \mathrm{T}-\mathrm{m}$ 8). Differentially enriched KO modules were identified according to their $\mathrm{W}$ statistic, from Wilcoxon rank-sum test which was performed on all the KOs at day 30.

\section{Results}

\section{L. casei significantly alleviates experimental arthritis}

SD rats were orally gavaged with or without the $L$. casei strain ATCC334 and subsequently challenged with complete Freund's adjuvant (CFA) on the same day (day 0) [13]. The animals were orally gavaged with $2 \times 10^{8} \mathrm{CFU}$ every day from day 0 to day 30 . As the culture medium for $L$. casei contains lactic acid and other metabolites, we washed off the medium, re-suspended the bacteria in $\mathrm{CMC}-\mathrm{Na}$, and then fed the live bacteria to the SD rats within half an hour. The activity of $L$. casei in CMC-Na was similar to that in MRS broth in such a short time
(Additional file 1: Figure S1, Additional file 2: Figure S2). MTX, a widely used DMARD [18], was used as a comparative control. After a 30-day intervention, the rats treated with $L$. casei or MTX showed less aggravated symptoms, as assessed by the arthritis scores and hind paw volumes (Fig. 1a, b), and rats treated with a combined therapy of $L$. case $i$ and MTX revealed an even better improvement than those with MTX or L. casei monotherapy (Additional file 3: Figure S3). Moreover, $L$. casei exerted its anti-arthritic effect without migrating to the places beside the gut as $L$. casei is facultative anaerobic bacteria and no bacteremia was detected in the $L$. casei-treated animals.

Bone and cartilage destruction, which often induce partial or permanent disability in poly-joints, are the major characteristics of pathological alterations in RA patients. Here, we used micro-computed tomography (micro-CT) to evaluate the effect of $L$. casei on bone remodeling. The bone erosion was diminished in the ankles of the $L$. casei-treated rats compared to the vehicletreated animals (Fig. 1c). Consistent with the micro-CT results, histological sections of the knees showed similar improvements after the intervention (Fig. 1d). In general, the pathological scores of the knees and the radiological scores of the ankles were significantly improved in the $L$. casei-treated animals compared to the vehicle-treated animals (Fig. 1e, g). The micro-CT score, calculated using the trabecular bone mineral density (BMD), bone volume rate $(\mathrm{BV} / \mathrm{TV})$, trabecular number $(\mathrm{Tb} . \mathrm{N})$, porosity percent (Po $\times$ total), and tissue mineral density (TMD), suggested severe osteoporosis in the vehicletreated animals (Additional file 4: Figure S4). Compared with the vehicle-treated rats, the rats treated with $L$. casei exhibited reduced bone erosion with a higher micro-CT score (Fig. 1f). From the three bone destruction evaluation scores, we created a classification scale that equally integrated the scores to more precisely assess the degree of damage. Using this classification scale, the $L$. casei-treated animals showed a significant change in the arthritis level from severe to moderate (Fig. 1h). Moreover, the metabolites produced by $L$. casei were also used separately in the AIA rats, and results were shown with no significant improvement (Additional file 3: Figure S3). These data indicate that administration of $L$. casei was able to alleviate experimental arthritis and protect bones from erosion.

\section{L. casei prevents gut dysbiosis caused by the induction of arthritis}

We next examined how the gut microbiota changed during the process of arthritis induction and how the administration of $L$. casei affected these changes. It is important to note that a well-defined RA-associated microbiota signature is lacking, so we collected fecal 

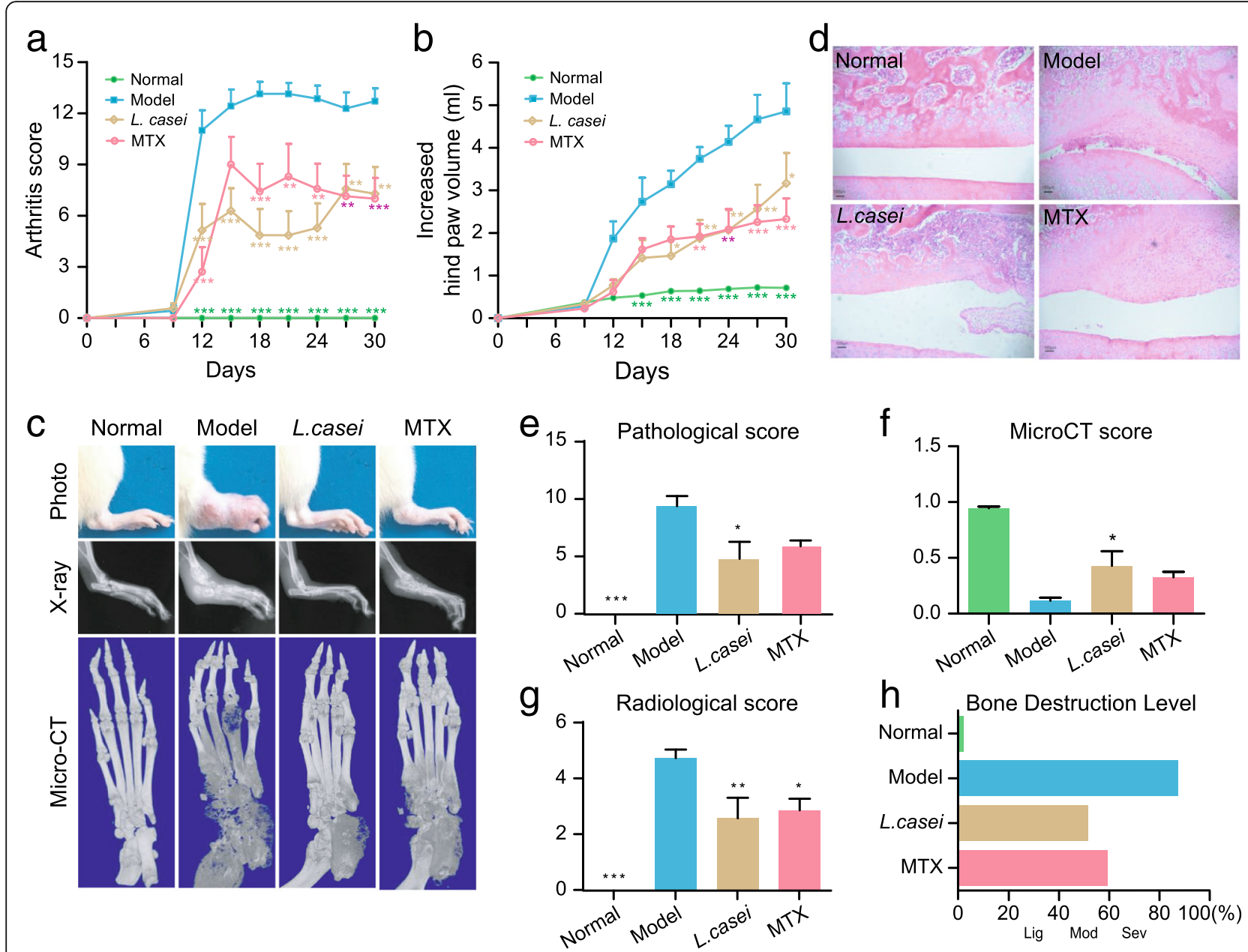

Fig. 1 L. casei alleviates the adjuvant-induced arthritis of rats. Effects of L. casei on arthritis score (a) and increased hind paw volume (b) are shown ( $n=7$ for each group). Data in $\mathbf{a}, \mathbf{b}$ are shown as mean \pm s.e.m. Differences between groups are analyzed by two-way ANOVA ( $* P<0.05$, ${ }^{* *} P<0.01,{ }^{* *} P<0.001$ VS model). The photographs, X-ray, and micro-CT images of ankles are shown in c. Representative images of pathological sections of knees in rats in different groups are shown in $\mathbf{d}$. The pathological improvements are assessed by pathological score $\mathbf{e}$. Radiological score and micro-CT score are evaluated using the micro-CT image and micro-CT analyzer, respectively $(\mathbf{f}, \mathbf{g})$. Data in $\mathbf{e}, \mathbf{f}$, and $\mathbf{g}$ are shown as mean \pm s.e.m. Differences among groups are analyzed by one-way ANOVA. ( ${ }^{*} P<0.05,{ }^{* *} P<0.01,{ }^{* * *} P<0.001$ VS model). The integral assessments of the bone destruction levels are shown in (h). Data are shown as mean and classified into several levels. 0-0.2: normal; 0.2-0.4: light (Lig); 0.4-0.6: moderate (Mod); 0.6-0.8: severe (Sev); 0.8 and above: very severe. Normal, normal control; model, disease control; MTX, methotrexate

samples from all four groups at five time points (TPs) and determined the dynamic alterations in the microbiome during the development of arthritis by using shotgun metagenomic sequencing. The five TPs included day 0 , before AIA induction (TP1), one pre-arthritis time point on day 7 (TP2), and three TPs, day 14 (TP3), day 21 (TP4), and day 30 (TP5), during arthritis progression in the AIA rats (Additional file 5: Figure S5).

To determine the dynamics of the changes in the gut microbiome composition in the $L$. casei ATCC334treated animals, we used a space-based calculation derived from the PCoA of the Bray-Curtis distances of the healthy and vehicle-treated subjects at the species level, which were set as the "healthy plane" (HP) and "disease plane" (DP), respectively. Distance from each sample to the HP and DP revealed dynamic changes in the gut microbiome, and the thickness of the line reflected the severity of arthritis. Samples from the rats treated with L. casei exhibited significant closer distances to the HP than the DP at TP2, while for the samples from the MTX-treated rats, there showed an apparent change at TP2 and a significant closer distance to DP than HP at TP3 (Fig. 2), suggesting that L. casei might remedy volatile gut dysbiosis at the early stage. At TP5, along with the relief of arthritis observed in L. casei group, the composition of the gut microbiomes was significantly closer to HP than DP (Fig. 2), suggesting that dysbiosis in arthritis could be reversed by $L$. casei, which finally 


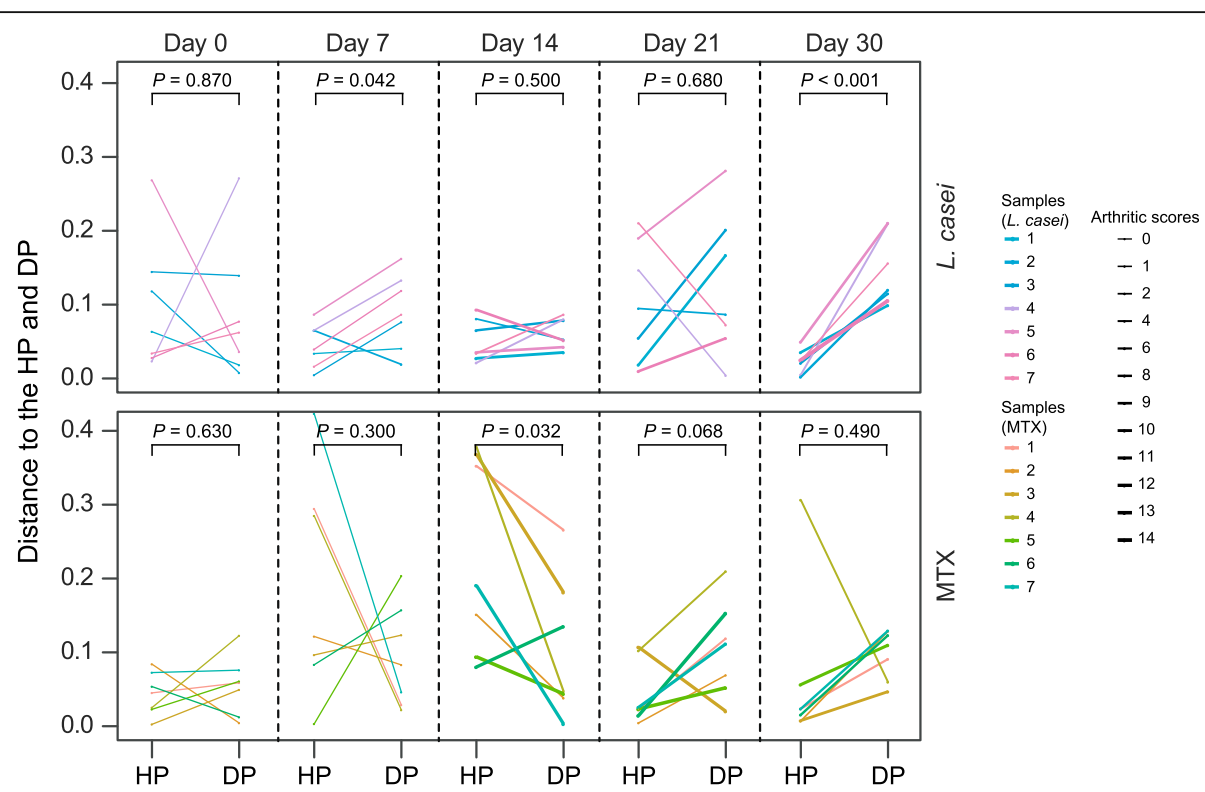

Fig. 2 Dynamic changes of the gut microbiota composition in the L. casei/MTX-treated rats over time. Distances from healthy plane (HP) and model plane (DP) for each sample of the L. casei-/MTX-treated rats on the five time points are shown. The colors of lines correspond to different samples and the thickness reflects the severity of arthritis. Difference between HP and DP is analyzed by paired $t$ tests

contributed to the amelioration of AIA. Interestingly, the arthritic scores were positively correlated with the distance to HP (Spearman's correlation coefficient = $0.48, P=0.015)$ and negatively correlated with the distance to DP (Spearman's correlation coefficient $=-0.46$, $P=0.019)$ after adjustment for weight and group, indicating that the alterations in microbiome are closely correlated to the severity of arthritis.

\section{L. casei remedies the perturbed microbiome in AIA rats}

To identify the microbial species or strains along with progression and treatment of arthritis, we next conducted a non-negative elastic-net regularized linear regression of the metagenomic sequencing results at the five TPs for each fecal sample against its related arthritis score. In total, 25 microbes were selected, among which Lachnospiraceae bacterium, Proteus mirabilis, and Corynebacterium urealyticum have been found to be related to RA $[7,19]$. The relative abundances of the identified 25 microbes were processed by normalization and then presented as $\log _{10}$ fold changes at each TPs in comparison to TP 1 . In the $\log _{10}$ fold change of TP2 to TP1, eight microbes were significantly altered in the model group, among which six microbes were changed in the MTX treated group, and only four were altered in $L$. casei treated rats (Additional file 6: Figure S6), consistent to the result that $L$. casei effectively inhibited RA progression at the very early stage, while at TP3, the alterations of microbes in L. casei group were much closer to those in model group (Additional file 7: Figure S7).
In the $\log _{10}$ fold change of TP5 to TP1, the relative abundances of Candidatus Arthromitus sp. SFB-rat Yit and Klebsiella pneumoniae were increased, while those of L. hominis, L. reuteri, and L. vaginalis were decreased after the induction of arthritis, indicating that these microbes may play important roles in the induction of experimental arthritis (Fig. 3). We further investigated the alterations of arthritis-correlated species in the $L$. casei-treated AIA rats. Unlike rats in the model group, a cluster of microbes including Acinetobacter unclassified, Corynebacterium casei, and L. acidophilus was upregulated (Fig. 3), and Corynebacterium urealyticum, Desulfovibrio desulfuricans, and Erysipelotrichaceae bacterium 21-3 were downregulated. Of note, these alterations occurred in the absence of persistent colonization by $L$. casei ATCC334, as we did not detect an enrichment of $L$. casei in the fecal samples isolated from the L. caseitreated rats (Additional file 8: Figure S8). However, the relative abundances of other Lactobacillus species were upregulated (such as L. acidophilus) or restored to the normal level ( such as the downregulated microbes in model group: L. hominis, L. reuteri, and L. vaginalis) (Fig. 3), suggesting that the function of $L$. casei in suppressing arthritis is closely associated with its function in modulating the microbiome, especially other Lactobacillus strains.

To determine whether the alterations in the microbiota were specific to $L$. casei, we further compared the differences in microbiome composition between the MTX- and L. casei-treated rats. The abundances of Erysipelotrichaceae bacterium 21-3, Corynebacterium 


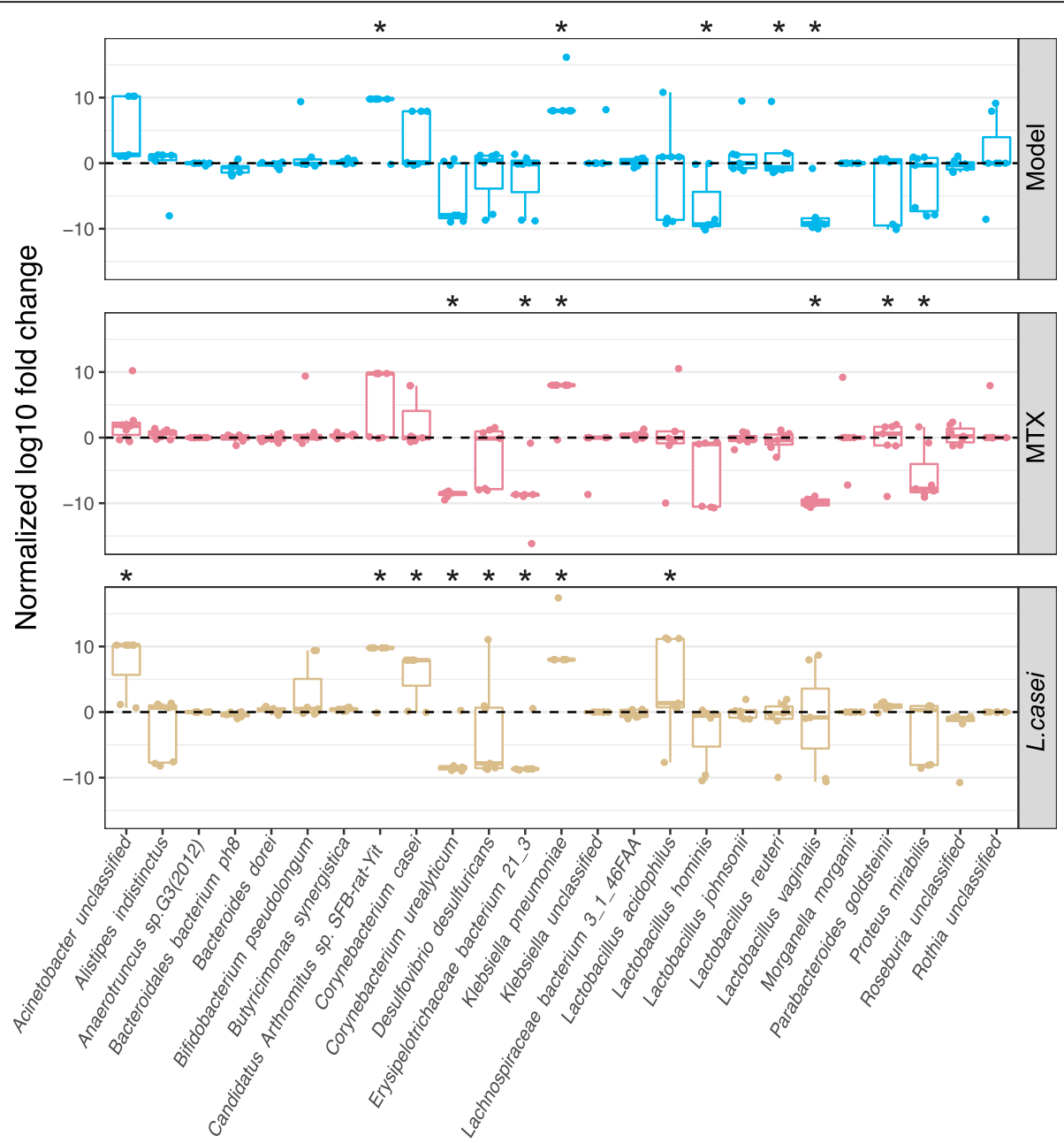

Fig. $3 \log _{10}$ fold change of the relative abundance of arthritis-correlated species at TP5 in comparison with samples of TP1. Boxes represent the median and interquartile ranges (IQRs) between the first and third quartiles; whiskers represent the lowest or highest values within 1.5 times IQR from the first or third quartiles. Circles represent samples. Significant fold change is marked with an asterisk

urealyticum, L. hominis, and L. reuteri were partly regulated by intervention with either of these two treatments (Fig. 3), which indicates that these microbes might be useful as biomarkers of therapeutic responses in RA patients. More interestingly, the abundances of Desulfovibrio desulfuricans, $L$. acidophilus, and $L$. vaginalis were effectively modulated by the $L$. casei treatment, but not by the MTX treatment. Thus, L. casei ATCC334 is capable of ameliorating arthritis by normalizing the levels of certain affected microbes in AIA rats.

\section{Inhibition of expression of pro-inflammatory cytokines correlates with the abundance of bacteria affected by administration of $L$. casei.}

Cytokines play critical roles in the pathogenesis of RA, while inhibiting expression or function of cytokines increases the probability of remission of RA and protects bones from destruction [20]. Therefore, we determined the serum levels of interferon- $\gamma$ (IFN- $\gamma)$, tumor necrosis factor- $\alpha$ (TNF- $\alpha$ ), and interleukins (IL)- $1 \beta$, IL-17, IL-2, and IL- 6 and determined the correlations between the levels of pro-inflammatory cytokines and alterations in the gut microbiota after $L$. casei treatment in AIA rats. The results showed a significant reduction in the levels of pro-inflammatory cytokines IFN- $\gamma$, TNF- $\alpha$, IL- $1 \beta$, IL17 , and IL-6 in the L. casei-treated AIA rats compared to the vehicle-treated AIA rats (Fig. 4a). In addition, the levels of IL-17 and IL-1 $\beta$, which are produced by activated adaptive and innate immune cells $[21,22]$, respectively, were significantly reduced in the animals treated with $L$. casei. Collectively, these data indicate that the alleviation of arthritis in the $L$. casei-treated rats was related to the suppression of pro-inflammatory cytokine expression.

To better understand the interaction between the abundances of the microbes associated with amelioration 

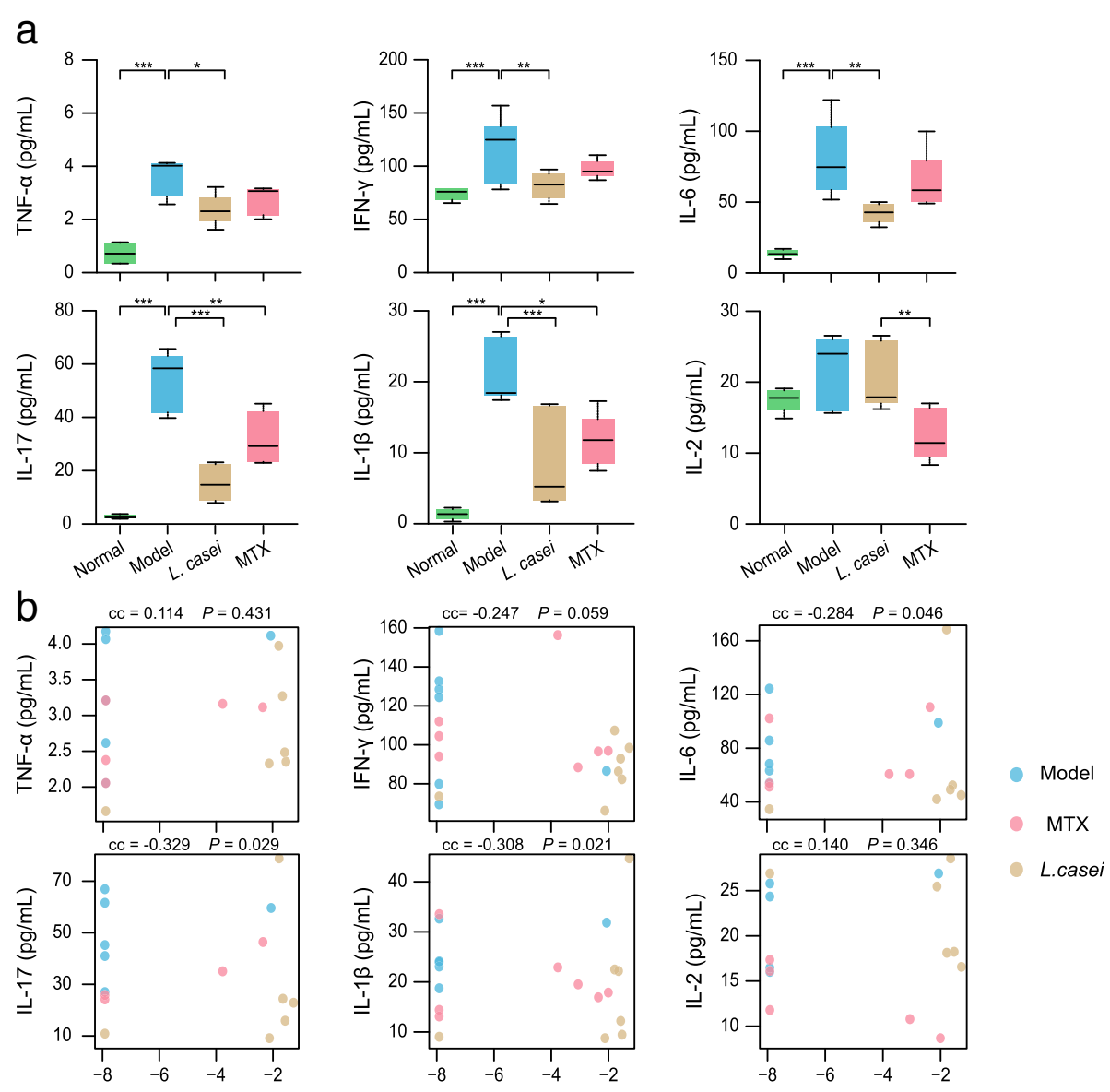

Lactobacillus acidophilus abundance $\left(\log _{10}\right)$

Fig. 4 L. casei inhibits pro-inflammatory cytokines expression via resurrection of L. acidophilus. a The expressions of cytokines (IL-17, IL-1 $\beta, T N F a$, IL-6, IFN- $\gamma$, IL-2) in serum are assessed using ELISA. Data are shown as mean \pm s.e.m and min, max. Differences between groups are analyzed by one-way ANOVA. $\left({ }^{*} P<0.05,{ }^{* *} P<0.01,{ }^{* * *} P<0.001\right.$ VS model). b Associations of the abundance of L. acidophilus with plasmatic cytokines. $C C$, Spearman's correlation coefficient after adjustment for weight, group, and arthritis score

of the AIA of rats and the expression levels of cytokines in the $L$. casei group, we computed covariances to analyze whether the relative abundance of $L$. casei or MTX treatment-correlated species (Erysipelotrichaceae bacterium 21-3, Corynebacterium urealyticum, Desulfovibrio desulfuricans, and Lactobacillus strains including $L$. hominis, $L$. acidophilus, $L$. reuteri, and $L$. vaginalis) were correlated with the expression of pro-inflammatory cytokines. Results showed that these bacteria were all significantly correlated to one or more inflammatory cytokines (Fig. 4b, Additional file 9: Figure S9, Additional file 10: Figure S10, Additional file 11: Figure S11, Additional file 12: Figure S12, Additional file 13: Figure S13, Additional file 14: Figure S14). Interestingly, most of the rats with enriched L. acidophilus in the L. casei-treated group showed decreased expression of pro-inflammatory cytokines and significant correlations were detected between the relative abundance of $L$. acidophilus and the expression level of IL-6,
IL-17, and IL-1 $\beta(P<0.05)$ (Fig. 4b). These results suggest that the effect of $L$. casei on AIA in rats is associated with the suppressive effect of treatment-correlated microbes on pro-inflammatory cytokine expression.

\section{Maintenance of the redox balance of oxidative stress is involved in the amelioration of RA in the AIA rats induced by treatment with $L$. casei}

To explore the potential mechanisms behind the beneficial effects of administration of $L$. casei, we conducted a comprehensive analysis of the functional modules in the gut microbiome of the L. casei- and MTX-treated rats using the KEGG database. Consistent with the notion that alterations in the microbiota lead to changes in host metabolism and the redox balance, the functional modules for the pentose phosphate pathway, including the oxidative phase, were found to be enhanced in the AIA rats, which was consistent with the results of previous 
reports on RA patients [23]. After treatment with $L$. casei, the abundance of genes involved in the pentose phosphate pathway was reduced, and the abundance of genes involved in the nicotinamide adenine dinucleotide (NADH) quinone oxidoreductase, assimilatory sulfate reduction, NADH ubiquinone oxidoreductase, beta-oxidation, and fatty acid biosynthesis modules were increased, suggesting that $L$. casei exerts its anti-arthritic effect through modulating immunometabolism and redox capacity (Fig. 5).

Moreover, $L$. acidophilus has been found to be able to improve the antioxidant status of collagen-induced arthritic rats [24]. These data imply that the modulation of the gut microbiome by $L$. casei, which results in the enrichment of L. acidophilus, is able to alleviate arthritis by maintaining the redox balance of oxidative stress.

\section{Discussion}

There is increasing evidence that using intensive DMARD-based strategies in early RA in the clinic is helpful for long-term remission and for achieving positive functional, radiographic, and prognostic outcomes $[25,26]$. The concept of a "window of opportunity" was proposed to emphasize the importance of early treatment for RA patients [27], but the strategy focusing on the triggering factors is limited. In the current study, we used a probiotic bacterium, $L$. casei, as a prophylactic treatment for arthritis. Because of its obvious effectiveness and low toxicity, supplementation with $L$. casei is probably an effective option to inhibit RA progression at the very early stage.

Previous studies have found dysbiosis in the gut microbiome of RA patients $[5,7,28]$, but little is known about the highly personalized microbiome dynamics

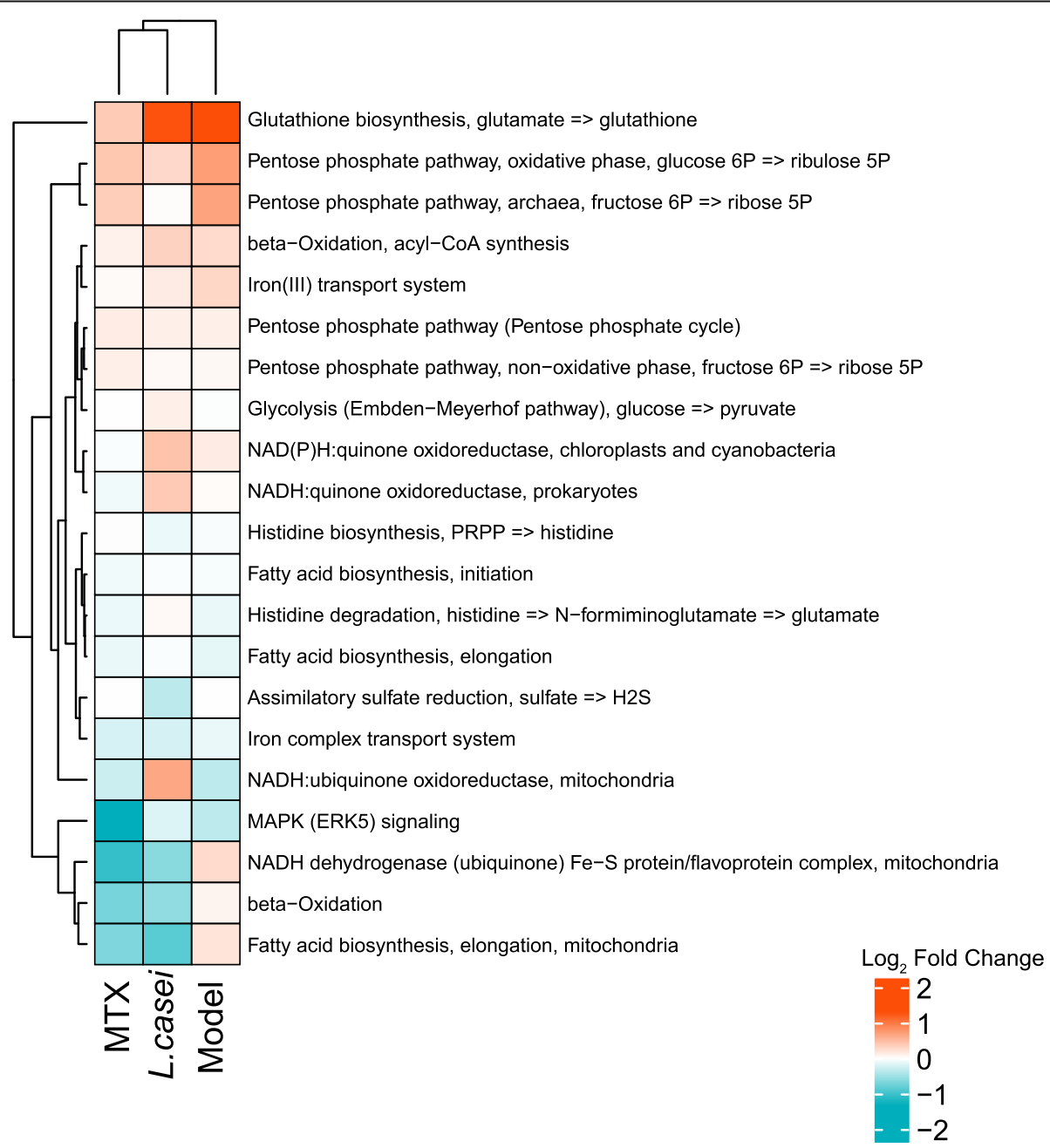

Fig. $5 \mathrm{~L}$. casei maintains the redox balance of oxidative stress. Mean $\log _{2}$ fold changes in the abundance of immune-related KEGG modules at TP5 in comparison with samples of TP1 from MTX, L. casei, and model group. The KEGG orthology group modules and groups are ordered by unsupervised hierarchical clustering. Cyan, reduced modules; red, increased modules. Modules missing from one or more groups are not plotted 
during the induction, progression, and treatment of arthritis, especially in regard to the entire genomes of all the organisms present. In the current study, we used shotgun metagenomic surveys and set an HP and DP to summarize the dynamics of all organisms for the first time. The results showed that $L$. casei, regardless of the function of the metabolites that produced by L. casei, was capable of maintaining the balance of the microbiome, along with the disease process, at a distance closer to the healthy state than to the disease state of the gut microbiome. These results indicate that $L$. casei could remedy the disordered microbiome caused by the induction of arthritis at the entire genome level, and the beneficial effects induced by the $L$. casei treatment might be attributed to specific alterations in the gut microbiome.

Therapeutic regimens that theoretically target the intestinal flora and the gut-joint axis have been in use since the 1940s [29]. Several regimens, such as sulfasalazine and minocycline [30], have been incorporated into DMARD regimens to treat RA, suggesting that an "optimal" microbiota is able to efficiently optimize immune responses [31]. In addition, probiotics have been shown to facilitate the efficacy of anticancer immunotherapies, such as anti-PD-L1 therapy, an immune checkpoint blockade approach [32, 33]. A previous study also showed that probiotics, such as $L$. casei 01 , might help alleviate the symptoms of RA and suppress pro-inflammatory cytokines in patients undergoing treatment with DMARDs [10, 34], suggesting that there is a positive synergistic effect between DMARDs and probiotics on arthritis, which was also confirmed in our study. Our current study showed that a significant reduction in the levels of pro-inflammatory cytokines was detected after 30 days of prophylactic treatment with $L$. casei. Though no enrichment of $L$. casei was detected in the fecal samples isolated from the $L$. casei-treated rats, the relative abundance of $L$. acidophilus, $L$. hominis, $L$. reuteri, and $L$. vaginalis were increased by the administration of $L$. case $i$ to the AIA rats, indicating that $L$. casei ameliorates arthritis mainly through rebalancing the Lactobacillus strains. Lactobacillus are the most prominent probiotics that are often present in the gastrointestinal tract. Some strains of Lactobacillus have been demonstrated to drive $\mathrm{T}$ cell differentiation from intraepithelial CD4+ $\mathrm{T}$ cells into immunoregulatory $\mathrm{T}$ (Treg) cells by generating indole derivatives of tryptophan that activate the arylhydrocarbon receptor of CD4+ T cells [35]. The metabolic products of these Lactobacillus strains, such as short-chain fatty acids, also promote the regulation of colonic Treg cell homeostasis [36, 37]. However, there remains to be confirmed that the difference of $L$. caseitriggered alterations of the microbiome in gastrointestinal tissues are the same to that in the fecal samples as is reported before [38], so as to draw a conclusion that
L. casei exerts its anti-arthritis effect via modulation of Lactobacillus strains.

In addition, we found that $L$. casei could markedly increase the L. acidophilus population in the gut microbiota of the AIA rats, which is in line with previous reports on L. acidophilus [39, 40]. Although the mechanism by which $L$. acidophilus modulates $\mathrm{T}$ helper (Th) 17 cell differentiation and production in the gut remains unclear, the downregulation of signal transducer and activator of transcription (STAT) 3 transcription [41] is one of the main molecular mechanisms by which $L$. acidophilus inhibits the IL-23/Th17 axis. Furthermore, L. acidophilus was confirmed to inhibit bone loss and increase bone heterogeneity in osteoporotic mice, suggesting that the protective effect of $L$. casei on the bones of AIA rats might partly be attributed to the modulation of L. acidophilus.

Reactive oxygen species (ROS) were initially regarded as merely damaging agents but now are recognized as second messengers that regulate cellular function through oxidant signaling [42]. The targeting of ROS generation is developing into a potential therapeutic approach for RA patients [43]. Naïve T cells from RA patients demonstrated that the replenishment of intracellular ROS corrected abnormal proliferative behavior in $\mathrm{T}$ cells and successfully suppressed synovial inflammation [23]. Modulating the redox balance and gut microbiome at the entire genome level might be involved in the anti-arthritic effect of $L$. casei on AIA rats [44]. The restored microbiome modulated by $L$. casei suppressed the activated pentose phosphate pathway and improved the NADH quinone oxidoreductase, assimilatory sulfate reduction, NADH ubiquinone oxidoreductase, beta-oxidation, and fatty acid biosynthesis modules. Lactobacilli show a distinct capacity for inducing cellular ROS generation [45]. Moreover, intestinal epithelial cells that came into contact with lactobacilli exhibited increased protein oxidation, resulting in the induction of the transcription of redox-stimulated modules, such as the nuclear factor (erythroid-derived 2)-like 2 (Nrf2) pathway [46]. Thus, restoring oxidant signaling via the modulation of the gut microbiome by $L$. casei may suppress arthritis through the suppression of pro-arthritogenic $\mathrm{T}$ cell effector functions.

The effects of $L$. casei in the occurrence, development, and treatment of arthritis are still not fully understood. We anticipate that large-scale studies will be focused on the potential roles of gut microbiome on RA, especially for the early diagnosis and early treatment of disease.

\section{Conclusions}

This current work is the first to identify the compositional and functional alterations in the gut microbiome by using a time-series research protocol in AIA rats, and 
the results support the conclusion that modulating the microbiota could be a new strategy for the prevention and treatment of human RA. Of note, these results were obtained in an experimental arthritis rat model, and there is a strong need to perform intensive investigations in RA patients. The precise molecular mechanisms behind the preventive/therapeutic actions of probiotics must be further elucidated, and the identified bacterial markers in the gut need to be intensively validated.

\section{Additional files}

Additional file 1: Figure S1. The differences of optical density in the concentration of $L$. casei pretreated by CMC-Na or MRS. (PDF $17 \mathrm{~kb}$ )

Additional file 2: Figure S2. The growth states of CMC-Na or MRS pretreated L. casei after culturing for $48 \mathrm{~h}$ in MRS agar. (PDF $29 \mathrm{~kb}$ )

Additional file 3: Figure S3. The arthritic scores in AIA rats. (PDF $54 \mathrm{~kb}$ ) Additional file 4: Figure S4. The alterations of trabecular bone mineral density (BMD), bone volume rate (BV/TV), trabecular number (Tb.N), porosity percent $(\mathrm{Po} \times$ total) and tissue mineral density (TMD) tested by Micro CT. (PDF $41 \mathrm{~kb}$ )

Additional file 5: Figure S5. The source of fecal samples for assessment the alterations of gut microbiome in AIA rats. (PDF $412 \mathrm{~kb}$ )

Additional file 6: Figure S6. The $\log _{10}$ fold change of the relative abundance of arthritis-correlated species at TP2 in comparison with samples of TP1. (PDF $205 \mathrm{~kb}$ )

Additional file 7: Figure S7. The $\log _{10}$ fold change of the relative abundance of arthritis-correlated species at TP3 in comparison with samples of TP1. (PDF $206 \mathrm{~kb}$ )

Additional file 8: Figure S8. Abundance of L. casei among fecal samples of different group. (PDF $1030 \mathrm{~kb}$ )

Additional file 9: Figure S9. Associations of the abundance of $D$. desulfuricans with plasmatic cytokines. (PDF $147 \mathrm{~kb}$ )

Additional file 10: Figure S10. Associations of the abundance of Enysipelotrichaceae bacterium 21_3 with plasmatic cytokines. (PDF 149 kb)

Additional file 11: Figure S11. Associations of the abundance of Corynebacterium urealyticum with plasmatic cytokines. (PDF 209 kb)

Additional file 12: Figure S12. Associations of the abundance of L. hominis with plasmatic cytokines. (PDF $148 \mathrm{~kb}$ )

Additional file 13: Figure S13. Associations of the abundance of L. reuteri with plasmatic cytokines. (PDF $142 \mathrm{~kb}$ )

Additional file 14: Figure S14. Associations of the abundance of L. vaginalis with plasmatic cytokines. (PDF $145 \mathrm{~kb}$ )

Additional file 15: Table S1. Metagenomic sequencing data for the SD rat microbiome. (XLSX $19 \mathrm{~kb})$

Additional file 16: Table S2. Relative abundances of species determined by the metagenomic sequencing. (XLSX $35 \mathrm{~kb}$ )

\section{Abbreviations}

AIA: Adjuvant-induced arthritis; BMD: Trabecular bone mineral density; BV/ TV: Bone volume rate; CFA: Complete Freund's adjuvant; CFU: Colonyforming units; CMC-Na: Sodium carboxymethyl cellulose; D. desulfuricans: Desulfovibrio desulfuricans; DMARDs: Disease-modifying antirheumatic drugs; DP: Disease plane; ELISA: Enzyme-linked immunosorbent assay; ENA: European nucleotide archive; ESR: Erythrocyte sedimentation rate; HP: Healthy plane; ICSH: International Council for Standardization in Haematology; IFN- - : Interferon- $\gamma$; IL: Interleukin; KEGG: Kyoto Encyclopedia of Genes and Genomes; L. casei: Lactobacillus casei; MHC: Major histocompatibility complex; micro-CT: Micro-computed tomography; MTX: Methotrexate; NADH: Nicotinamide adenine dinucleotide; Nrf2: Nuclear factor (erythroid-derived 2)-like 2; PCoA: Principal coordinates analysis; Po $\times$ total: Porosity percent; RA: Rheumatoid arthritis; ROS: Reactive oxygen species; SD: Sprague-Dawley; SE: Single-end; STAT: Signal transducer and activator of transcription; Tb.N: Trabecular number; Th: T helper; TMD: Tissue mineral density; TNF-a: Tumor necrosis factor-a; TP: Time point;

Treg: Regulatory $\mathrm{T}$

\section{Acknowledgements}

We gratefully acknowledge the assistance and guidance of Karsten Kristiansen from BGI-Shenzhen.

\section{Authors' contributions}

HDP carried out the experimental work, participated in the design of experiments, and drafted the manuscript. RJG, YMJ, and QW carried out the bioinformatics analyses and helped to draft the manuscript. YX, YFZ, and RZL participated in the conduction of the animal study. ZQL, LLL, TL, and LHL participated in the design of animal experiments. JZ, FL, LX, and BT helped to collect the sample and do the statistical analysis. XX, JW, HMY, and HZ revised the manuscript. LL and HJJ designed the experiments, acquired the funding, and revised the manuscript. The final manuscript was read and approved by all the authors.

\section{Funding}

This work was financially supported by grants from the Macau Science and Technology Development Fund (102/2016/A3) and the Shenzhen Municipal Government of China (JSGG20160229172752028, JCYJ20160229172757249).

\section{Availability of data and materials}

Metagenomic sequencing data for all samples have been deposited in the European Nucleotide Archive (ENA) database under the accession identification number PRJEB22973. Other relevant data have been deposited in the GigaScience Database (GigaDB, http://gigadb.org/dataset/100440).

Ethics approval and consent to participate

All experimental procedures were conducted in compliance with the institutional guidelines for the care and use of laboratory animals in China and approved by the Animal Ethical Council of Guangzhou University of Chinese Medicine. Animal welfare and experimental procedures were strictly in accordance with the guidelines for the care and use of laboratory animals.

\section{Consent for publication}

Not applicable.

\section{Competing interests}

The authors declare that they have no competing interests.

\section{Author details}

${ }^{1}$ State Key Laboratory of Quality Research in Chinese Medicine/Macau Institute for Applied Research in Medicine and Health, Macao University of Science and Technology, Macao, China. ${ }^{2}$ BGI-Shenzhen, Shenzhen 518083, China. ${ }^{3}$ China National Genebank, BGI-Shenzhen, Shenzhen 518120, China. ${ }^{4}$ International Institute for Translational Research of Traditional Chinese Medicine of Guangzhou University of Chinese Medicine, Guangzhou 510006, Guangdong, China. ${ }^{5}$ Fujian University of Traditional Chinese Medicine, No.1, Qiuyang Road, Minhoushangjie, Fuzhou 350122, Fujian, China. ${ }^{6}$ BGl Education Center, University of Chinese Academy of Sciences, Shenzhen 518083, China. ${ }^{7}$ Shenzhen Engineering Laboratory of Detection and Intervention of human intestinal microbiome, BGl-Shenzhen, Shenzhen 518083, China. ${ }^{8}$ James D. Watson Institute of Genome Sciences, Hangzhou 310058, China.

Received: 23 January 2019 Accepted: 3 July 2019

Published online: 17 July 2019

\section{References}

1. Firestein GS, McInnes IB. Immunopathogenesis of rheumatoid arthritis. Immunity. 2017;46(2):183-96.

2. Scott DL, Wolfe F, Huizinga TW. Rheumatoid arthritis. Lancet. 2010; 376(9746):1094-108.

3. Raza K, et al. Treating very early rheumatoid arthritis. Best Pract Res Clin Rheumatol. 2006;20(5):849-63.

4. Schett G, Gravallese E. Bone erosion in rheumatoid arthritis: mechanisms, diagnosis and treatment. Nat Rev Rheumatol. 2012;8(11):656-64. 
5. Scher JU, Abramson SB. The microbiome and rheumatoid arthritis. Nat Rev Rheumatol. 2011;7(10):569-78.

6. Kubinak JL, et al. MHC variation sculpts individualized microbial communities that control susceptibility to enteric infection. Nat Commun. 2015;6:8642

7. Zhang $X$, et al. The oral and gut microbiomes are perturbed in rheumatoid arthritis and partly normalized after treatment. Nat Med. 2015;21(8):895-905.

8. Rashid T, Ebringer A. Autoimmunity in rheumatic diseases is induced by microbial infections via crossreactivity or molecular mimicry. Autoimmune Dis. 2012;2012:539282.

9. Pan $\mathrm{H}$, et al. A gene catalogue of the Sprague-Dawley rat gut metagenome. Gigascience. 2018;7(5):1-8.

10. Alipour B, et al. Effects of Lactobacillus casei supplementation on disease activity and inflammatory cytokines in rheumatoid arthritis patients: a randomized double-blind clinical trial. Int J Rheum Dis. 2014;17(5):519-27.

11. Aktas B, et al. The impact of lactobacillus casei on the composition of the cecal microbiota and innate immune system is strain specific. PloS One. 2016;11(5):e0156374

12. Adan $\mathrm{N}$, et al. Prolactin promotes cartilage survival and attenuates inflammation in inflammatory arthritis. J Clin Invest. 2013;123(9):3902-13.

13. Cai $X$, et al. Manipulation of the induction of adjuvant arthritis in SpragueDawley rats. Inflamm Res. 2006;55(9):368-77.

14. Huang J, et al. A reference human genome dataset of the BGISEQ-500 sequencer. Gigascience. 2017;6(5):1-9.

15. Truong DT, et al. MetaPhIAn2 for enhanced metagenomic taxonomic profiling. Nat Methods. 2015;12(10):902-3.

16. Halfvarson J, et al. Dynamics of the human gut microbiome in inflammatory bowel disease. Nat Microbiol. 2017;2:17004.

17. Karlsson FH, et al. Gut metagenome in European women with normal, impaired and diabetic glucose control. Nature. 2013;498(7452):99-103.

18. Smolen JS, et al. EULAR recommendations for the management of rheumatoid arthritis with synthetic and biological disease-modifying antirheumatic drugs: 2016 update. Ann Rheum Dis. 2017;76(6):960-77.

19. Christopoulos $\mathrm{G}$, et al. Greek rheumatoid arthritis patients have elevated levels of antibodies against antigens from Proteus mirabilis. Clin Rheumatol. 2017;36(3):527-35.

20. Gravallese EM. Bone destruction in arthritis. Ann Rheum Dis. 2002;61(Suppl 2):ii84-6.

21. Gaffen SL, et al. The IL-17 cytokine family. Vitam Horm. 2006;74:255-82.

22. Schett G, Dayer JM, Manger B. Interleukin-1 function and role in rheumatic disease. Nat Rev Rheumatol. 2016;12(1):14-24.

23. Yang $Z$, et al. Restoring oxidant signaling suppresses proarthritogenic $T$ cell effector functions in rheumatoid arthritis. Sci Transl Med. 2016;8(331):331ra38.

24. Amdekar S, Singh V. Lactobacillus acidophilus maintained oxidative stress from reproductive organs in collagen-induced arthritic rats. J Hum Reprod Sci. 2016;9(1):41-6.

25. Monti $S$, et al. Rheumatoid arthritis treatment: the earlier the better to prevent joint damage. RMD Open. 2015;1(Suppl 1):e000057.

26. Mottonen $\mathrm{T}$, et al. Comparison of combination therapy with single-drug therapy in early rheumatoid arthritis: a randomised trial. FIN-RACo trial group. Lancet. 1999;353(9164):1568-73.

27. Raza K, Filer A. The therapeutic window of opportunity in rheumatoid arthritis: does it ever close? Ann Rheum Dis. 2015;74(5):793-4.

28. Maeda Y, Kumanogoh A, Takeda K. Altered composition of gut microbiota in rheumatoid arthritis patients. Nihon Rinsho Men'eki Gakkai Kaishi. 2016;39(1):59-63.

29. Brusca SB, Abramson SB, Scher JU. Microbiome and mucosal inflammation as extra-articular triggers for rheumatoid arthritis and autoimmunity. Curr Opin Rheumatol. 2014;26(1):101-7.

30. Zheng $\mathrm{H}$, et al. Modulation of Gut microbiome composition and function in experimental colitis treated with sulfasalazine. Front Microbiol. 2017:8:1703.

31. Belkaid $Y$, Hand TW. Role of the microbiota in immunity and inflammation. Cell. 2014;157(1):121-41.

32. Vetizou $M$, et al. Anticancer immunotherapy by CTLA-4 blockade relies on the gut microbiota. Science. 2015:350(6264):1079-84.

33. Sivan $\mathrm{A}$, et al. Commensal Bifidobacterium promotes antitumor immunity and facilitates anti-PD-L1 efficacy. Science. 2015:350(6264):1084-9.

34. Pan $\mathrm{H}$, et al. Whether probiotic supplementation benefits rheumatoid arthritis patients: a systematic review and meta-analysis. Engineering. 2017;3(1):115-21.
35. Cervantes-Barragan $L$, et al. Lactobacillus reuteri induces gut intraepithelia CD4(+)CD8alphaalpha(+) T cells. Science. 2017:357(6353):806-10.

36. Smith PM, et al. The microbial metabolites, short-chain fatty acids, regulate colonic Treg cell homeostasis. Science. 2013;341(6145):569-73.

37. Arpaia N, et al. Metabolites produced by commensal bacteria promote peripheral regulatory T-cell generation. Nature. 2013;504(7480):451-5.

38. Zoetendal EG, et al. Mucosa-associated bacteria in the human gastrointestinal tract are uniformly distributed along the colon and differ from the community recovered from feces. Appl Environ Microbiol. 2002;68(7):3401-7.

39. Chen $L$, et al. Lactobacillus acidophilus suppresses colitis-associated activation of the IL-23/Th17 axis. J Immunol Res. 2015;2015:909514.

40. Park JS, et al. Lactobacillus acidophilus improves intestinal inflammation in an acute colitis mouse model by regulation of Th17 and Treg cell balance and fibrosis development. J Med Food. 2018:21(3):215-24.

41. Owaga $E$, et al. Th17 Cells as potential probiotic therapeutic targets in inflammatory bowel diseases. Int J Mol Sci. 2015;16(9):20841-58.

42. Holmstrom KM, Finkel T. Cellular mechanisms and physiological consequences of redox-dependent signalling. Nat Rev Mol Cell Biol. 2014;15(6):411-21.

43. Fan XX, et al. Suppression of lipogenesis via reactive oxygen species-AMPK signaling for treating malignant and proliferative diseases. Antioxid Redox Signal. 2018;28(5):339-57.

44. Fan XX, et al. Novel therapeutic strategy for cancer and autoimmune conditions: modulating cell metabolism and redox capacity. Pharmacol Ther. 2018:191:148-61.

45. Jones RM, Neish AS. Redox signaling mediated by the gut microbiota. Free Radic Biol Med. 2017:105:41-7.

46. Jones RM, et al. Lactobacilli modulate epithelial cytoprotection through the Nrf2 pathway. Cell Rep. 2015;12(8):1217-25.

\section{Publisher's Note}

Springer Nature remains neutral with regard to jurisdictional claims in published maps and institutional affiliations.

Ready to submit your research? Choose BMC and benefit from:

- fast, convenient online submission

- thorough peer review by experienced researchers in your field

- rapid publication on acceptance

- support for research data, including large and complex data types

- gold Open Access which fosters wider collaboration and increased citations

- maximum visibility for your research: over $100 \mathrm{M}$ website views per year

At BMC, research is always in progress.

Learn more biomedcentral.com/submission 